\title{
What is the role of sentinel lymph node biopsy in the management of oral cancer in 2020 ?
}

\author{
Remco de Bree ${ }^{1}(\mathbb{0})$ Bart de Keizer ${ }^{2}(1) \cdot$ Francisco J. Civantos $^{3} \cdot$ Robert P. Takes $^{4} \cdot$ Juan P. Rodrigo $^{5}$.

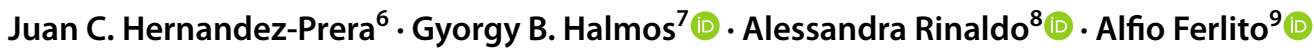

Received: 28 September 2020 / Accepted: 30 November 2020 / Published online: 28 December 2020

(c) The Author(s) 2020

\begin{abstract}
Approximately $70-80 \%$ of patients with cT1-2N0 oral squamous cell carcinoma (OSCC) ultimately prove to have no cancer in the cervical lymphatics on final pathology after selective neck dissection. As a result, sentinel lymph node biopsy (SLNB) has been adopted during the last decade as a diagnostic staging method to intelligently identify patients who would benefit from formal selective lymphadenectomy or neck irradiation. While not yet universally accepted, SLNB is now incorporated in many national guidelines. SLNB offers a less invasive alternative to elective neck dissection (END), and has some advantages and disadvantages. SLNB can assess the individual drainage pattern and, with step serial sectioning and immunohistochemistry (IHC), can enable the accurate detection of micrometastases and isolated tumor cells (ITCs). Staging of the neck is improved relative to END with routine histopathological examination. The improvements in staging are particularly notable for the contralateral neck and the pretreated neck. However, for floor of mouth (FOM) tumors, occult metastases are frequently missed by SLNB due to the proximity of activity from the primary site to the lymphatics (the shine through phenomenon). For FOM cancers, it is advised to perform either elective neck dissection or superselective neck dissection of the preglandular triangle of level I. New tracers and techniques under development may improve the diagnostic accuracy of SLNB for early-stage OSCC, particularly for FOM tumors. Treatment of the neck (either neck dissection or radiotherapy), although limited to levels I-IV, remains mandatory for any positive category of metastasis (macrometastasis, micrometastasis, or ITCs). Recently, the updated EANM practical guidelines for SLN localization in OSCC and the surgical consensus guidelines on SLNB in patients with OSCC were published. In this review, the current evidence and results of SLNB in early OSCC are presented.
\end{abstract}

Keywords Sentinel node biopsy $\cdot$ Oral cancer $\cdot$ Lymph node metastasis $\cdot$ Staging $\cdot$ Neck dissection

This article was written by members and invitees of the International Head and Neck Scientific group (www.IHNSG.com).

Remco de Bree

r.debree@umcutrecht.nl

1 Department of Head and Neck Surgical Oncology, University Medical Center Utrecht, P.O. Box 85500, 3508 GA Utrecht, The Netherlands

2 Department of Radiology and Nuclear Medicine, University Medical Center Utrecht, Utrecht, The Netherlands

3 Department of Otolaryngology, Sylvester Cancer Center, University of Miami, Florida, USA

4 Department of Otolaryngology/Head and Neck Surgery, Radboud University Medical Center, Nijmegen, The Netherlands
5 Department of Otolaryngology, Hospital Universitario Central de Asturias-University of Oviedo, ISPA, IUOPA, Oviedo, Spain

6 Department of Pathology, Moffitt Cancer Center, Tampa, FL, USA

7 Department of Otolaryngology/Head and Neck Surgery, University Medical Center Groningen, Groningen, The Netherlands

8 Department of Otolaryngology, University of Udine School of Medicine, Udine, Italy

9 International Head and Neck Scientific Group, Padua, Italy 


\section{Introduction}

Cervical lymph node metastasis is the single most important prognostic factor in oral squamous cell carcinoma (OSCC), and accurate detection of cervical lymph node metastases is critical for surgical and adjuvant therapy planning. Palpation has a low sensitivity in detecting lymph node metastases and proved to be inferior compared to conventional imaging techniques, such as ultrasonography (US), computerized tomography (CT) and magnetic resonance imaging (MRI) and Fluorine-18-fluorodeoxyglucose positron emission tomography (FDG PET). US, $\mathrm{CT}$ and MRI imaging modalities focus on morphologic (size and homogeneity) aspects of lymph nodes and FDG PET focusses on glucose consumption. None of these imaging techniques is able to detect small or microscopic subclinical metastases and to subsequently change treatment strategies.

US-guided fine-needle aspiration cytology (USgFNAC) shows a higher sensitivity and higher specificity than CT, MRI and FDG PET, but it also has its limitations. USgFNAC is strongly dependent on the experience and skills of the ultrasonographer and cytopathologist. Moreover, sensitivity will always be limited due to the inability to detect small metastatic deposits and to eliminate sampling error. Taking into account the limitations of palpation and imaging, patients with a clinically and radiologically negative neck (cN0) still have a substantial risk of having occult metastases in the neck [1].

How to treat patients with a substantial risk of occult metastases has been a heavily discussed topic for decades, especially in surgically treated patients who do not need an approach of the neck as part of management of the primary tumor. In early-stage OSCC (cT1-T2N0), it is usually not necessary to access the neck when the primary tumor can be resected transorally and no reconstruction is necessary. However, the incidence of occult metastases is reported to be around 30\% in these cases. A debate has been ongoing for many years on how to manage patients with a clinically negative neck: should all patients be treated prophylactically with an elective neck dissection, or can the neck be left untreated with a "watchful waiting" follow-up policy [2]. SLNB provides an intermediate approach between these two options, allowing for the selection of truly positive patients for further treatment.

Sentinel lymph node biopsy (SLNB) is a diagnostic staging procedure, which is nowadays also performed in OSCC. According the sentinel node concept, the first draining lymph nodes, the sentinel lymph nodes (SLNs), are most likely to harbor metastases in case of lymphogenic tumor spread and has to be identified and extirpated. The histopathological status of the SLN reflects the status of the rest of the nodal basin, and additional treatment (surgery and/or radiotherapy) of the nodal basin should be done if metastatic tumor deposits are found in the SLN. However, if the SLN is negative neck, no treatment of the nodal basin is needed and watchful waiting of the nodal basin is justified [3].

In OSCC, the concept of SLNB has evolved during the last decades. The first studies on visualization of the cervical lymphatic vessels were reported in the 1960s. In the 1980s, mapping of lymphatic drainage from specific head and neck sites was introduced. SLNB was further developed in the 1990s and after validation in this century, the procedure is now routinely performed in early OSCC in many head and neck centers. In short, the routine SLN procedure usually consists of preoperative peritumoral injections of a technetium-99 m ( $\left.{ }^{99 \mathrm{~m}} \mathrm{Tc}\right)$-labelled nanocolloid, or other radiotracer, followed by lymphoscintigraphy using planar and single photon emission computed tomography/CT (SPECT-CT) imaging. Based on the lymphoscintigraphy, the position of the SLN is marked on the skin. Subsequently the marked SLN is surgically removed, while intraoperative detection is achieved using a portable gamma probe. Finally, meticulous histopathological examination, including step serial sectioning and immunohistochemistry, of the harvested SLN is performed [3].

In 2010, we provided an update on the role of SLNB in the management of oral cancer [4]. Now, 10 years later, we want to update the knowledge on this topic by discussing the recent developments and the current role of SNLB in early OSCC.

\section{Sentinel lymph node biopsy as alternative for elective neck dissection}

Since 2010, large single-center and multicenter studies in which treatment of the neck was only performed after positive SLNs showed a high sensitivity and negative predictive value. Because false positive results are unlikely to occur, specificity and positive predictive value will always be near $100 \%$. To assess the diagnostic value of SLNB for the detection of occult lymph node metastases, follow-up of the untreated neck is a better reference standard than histopathological examination since missed lymph node metastases (false negative SNLB findings) will progress into clinically manifest ones and routine histopathological examination (not using step-serial sectioning with immunohistochemistry) of an END (irrespective of the SLNB result) may miss micrometastases (defined as tumor clusters $>0.2 \mathrm{~mm}$ and $<2 \mathrm{~mm}$ in size) and isolated tumor cells (ITC; defined as small clusters of cells not larger than $0.2 \mathrm{~mm}$, or single cells, or fewer than 200 cells in a single cross section) up to $15 \%$ [5]. Furthermore, in analyzing the accuracy of SLNB, 
it is important to realize that incidence of occult lymph node metastases and follow-up time (as reference standard) affect the negative predictive value (NPV) and sensitivity, respectively. The lower the incidence, the more likely the NPV is higher. The shorter the follow-up time, the lower the incidence of delayed (missed) metastases (recurrences) and the higher the sensitivity may be.

\section{Single-center studies}

Considering that histopathology of SLN-positive patients in combination with long-term follow-up of SLN-negative patients is the best available reference standard to define the accuracy of the SLNB procedure, we will discuss studies which used this reference standard (Table 1). Broglie et al. performed a prospective consecutive cohort analysis of 111 cT1-2N0 OSCC patients with an incidence of $40 \%$ occult lymph node metastases and found a sensitivity of $93 \%$ and NPV of $96 \%$ after a median follow-up of 37 months. Lymph node mapping consisted of preoperative dynamic and static lymphoscintigraphy and additional SPECT-CT in 34\% of cases. They demonstrated that even small tumor deposits (ITC and micrometastasis), only detectable by the extensive histopathologic workup of the SLNB protocol, have a significant impact on tumor control and survival [6]. Den Toom et al. reported on the results of SLNB in 90 cT1-2N0 OSCC patients: sensitivity and NPV were $93 \%$ and $97 \%$, respectively. The median follow-up was 18 months. In $65 \%$ of patients, SPECT/CT was performed preoperatively and additionally in all patients intraoperatively patent blue dye was peritumorally injected for visualization (coloring) of SLNs [7]. In the largest single center study of 253 cT1-2N0 OSCC patients of whom $30 \%$ had lymph node metastases Pedersen et al. using a median follow-up of 32 months as reference standard found for SNLB a sensitivity and NPV of $88 \%$, and $95 \%$, respectively. SPECT-CT was used in all patients. In their series, the group of Copenhagen did not yet use near-infrared (NIR) fluorescence imaging to improve the SNB procedure by facilitating intraoperative visual identification of the SLN [8]. Moya-Plana et al. reported on SNLB in 179 cT1-2N0 OSCC patients with a long term (median 5.3 years) follow-up who underwent neck dissection only if a SLN was positive and reported a sensitivity of $80 \%$ and NPV of $95 \%$. This lower sensitivity may be due to the high number (40\%) of floor of mouth tumors (see below) and long follow-up time. SPECT-CT was not used in all patients. Frozen section of SLN was used intraoperatively to perform a completion (therapeutic) neck dissection in the same session in case of a positive SLN [9]. Figures about the sensitivity of frozen sections to detect occult metastasis in SLNs were not reported. Due to tissue processing limitations and artifacts, frozen section analysis of SLNs can underestimate the true incidence of metastasis, particularly in the identification of ITC and micrometastasis. False negative SLNs may contain tiny deposits of microscopic metastatic disease, which may be missed in the examined histologic slides. Vorburger et al. compared the detection rate of occult metastases by monoslice frozen section with the definitive histopathologic work up. They found a sensitivity of frozen section of $47 \%$ which was dependent on the metastases size: for ITC $8 \%$, for
Table 1 Results of largest studies on SLNB in OSCC patients with adequate reference standard

\begin{tabular}{lllllll}
\hline Study & Number & Incidence (\%) & Follow-up* & $\begin{array}{l}\text { Sensitiv- } \\
\text { ity (\%) }\end{array}$ & NPV (\%) & $\begin{array}{l}\text { FOM } \\
\text { tumors } \\
(\%)\end{array}$ \\
\hline Single center studies & & & & & & \\
Broglie et al. [6] & 111 & 40 & $37(2-83)$ & 93 & 96 & 26 \\
Den Toom et al. [7] & 90 & 33 & $18(2-62)$ & 93 & 97 & 26 \\
Pedersen et al. [8] & 253 & 27 & $32(1-92)$ & 88 & 95 & 47 \\
Moya-Plana et al. [9] & 179 & 27 & 62 & 80 & 95 & 40 \\
Mølstrøm et al. [11] & 220 & 30 & $30(3-111)$ & 83 & 93 & 31 \\
Boeve et al. [12] & 91 & 33 & $32(21-47)$ & 85 & 94 & 30 \\
Multicenter studies & & & & & & 36 \\
Flach et al. [13] & 62 & 40 & $53(5-77)$ & 80 & 88 & 36 \\
Schilling et al. [14] & 415 & 26 & 36 & 86 & 94 & 25 \\
Den Toom et al. [19] & 488 & 22 & $26(0-116)$ & 81 & 93 & 27 \\
Meta-analyses & & & & 87 & 94 & \\
Liu et al. [16] & 3566 & & & 92 & 96 & 0 \\
Yang et al. [17] & 1084 & & & & & \\
\hline
\end{tabular}

Incidence of lymph node metastasis

$N P V$ negative predictive value, FOM floor of mouth; percentages of included OSCC patients

*Median (range) follow-up in months 
micrometastases 43\%, and for macrometastases 93\% [10]. Mølstrøm et al. analyzed SLNB in 220 cT1-2N0 OSCC patients with an incidence of occult metastasis of $30 \%$ and found a sensitivity of $83.3 \%$ and a NPV of $93.3 \%$ after a median follow-up of 30 months. SPECT-CT was used in the majority of patients. The authors focused on the topographical distribution of sentinel nodes and metastases (see below) [11]. Boeve et al. found a sensitivity of $85 \%$ and NPV of $94 \%$ for SLNB in detecting occult metastases (incidence $33 \%$ ) in $91 \mathrm{cT} 12 \mathrm{~N} 0$ OSCC patients after a median follow-up of 32 months [12].

\section{Multicenter studies}

Since 2010 also, some multicenter studies have been published with slightly lower sensitivity and specificity as can be expected. Flach et al. reported on a Dutch multicenter study in $62 \mathrm{cT} 1-2 \mathrm{~N} 0$ OSCC patients a sensitivity was $80 \%$ and NPV $88 \%$. SLNB was able to reduce the risk of occult lymph node metastases in OSCC patients from 40 to $8 \%$, and enabled an excellent neck control (97.4\%) [13]. In the largest multicenter study of $415 \mathrm{cT} 12 \mathrm{~N} 0$ OSCC patients, a sensitivity of $86 \%$ and NPV of $95 \%$ were found for SNLB to detect occult lymph node metastasis (incidence 26\%) after 3-year follow-up [14]. In the latter European SENT trial, all participating centers had to complete at least ten successful training SNLB procedures (validated against neck dissection) prior to recruiting to SENT. In the Dutch SNUS trial, this learning curve was not a criterion for unit participation, which is likely the reason for the lower accuracy. The importance of the learning curve was already shown in the ACOSOG Z0360 study in which the NPV of procedures performed by surgeons who entered the trial with more experience in the use of SLNB for oral cancer was higher than for less experienced surgeons [15].

\section{Meta-analysis}

In the most recent meta-analyses, also a high sensitivity and NPV for SNLB to detect occult lymph node metastases in OSCC were found. In 1 meta-analysis comprising 66 studies (3566 OSCC patients) a pooled sensitivity of $87 \%$ and a pooled negative predictive value of $94 \%$ were found [16]. In another meta-analysis, 35 studies (with 1084 tongue squamous cell carcinoma patients) were included. The pooled overall sensitivity and NPV of SLNB were $92 \%$ and $96 \%$, respectively [17]. In these meta-analyses, studies which used routine histopathological examination of elective neck dissection specimens as a reference standard, were included, which may result in an overestimation of the accuracy because of possible false negative histopathological results [5].

\section{Comparison sentinel node biopsy and elective neck dissection}

Only a few studies have compared recurrence rates and survival after SLNB and END. Moya-Plana et al. compared patients who underwent systematic END $(n=50)$ to patients who underwent only neck dissection if SLNB was positive $(n=179)$. Nodal recurrence was reported in $10.0 \%$ and $7.8 \%$, respectively. They found no statistically significant difference between the systematic ND group and the SNB group for 5 -year recurrence free survival $(77.7 \%$ vs $80.7 \% ; p=0.84)$ and for overall survival $(78.7 \%$ and $76.4 \%$; $p=0.73$ ) [9]. In a recent retrospective cohort study of 8328 patients with cT1-2cN0 OCSCC who underwent staging of the neck by SLNB $(n=240 ; 2.9 \%)$ or END in the United States National Cancer Data Base from 2012 to 2015, with a median follow-up of 35.4 months, the overall 3-year survival was significantly better after SLNB $(82.0 \%)$ than after END $(77.5 \%, p=0.40)$. However, after adjustment, overall survival was equivalent between patients who underwent SLNB versus END (adjusted hazard ratio 1.03, confidence interval 0.67-1.59). Moreover, SLNB was associated with reduced perioperative morbidity, with median length of hospital stay of 1.0 days versus 3.0 days after $\operatorname{END}(p<0.001)$. Perioperative 30 -day mortality was $0 \%$ after SLNB versus $0.7 \%$ after a recent retrospective, multicenter cohort study included 390 patients staged by END and 488 by SLNB. The overall sensitivity ( $84 \%$ vs. $81 \%$ ) and NPV (both $93 \%$ ) were comparable between END and SLNB patients. The END cohort contained more pT2 tumors (51\%) compared to the SLNB cohort $(23 \%)(p<0.001)$. No differences were found for sensitivity and NPV between SLNB and END stratified for pT stages. In floor of mouth (FOM) tumors, SLNB had a lower sensitivity (63\% vs. $92 \%, p=0.006)$ and NPV $(90 \%$ vs. $97 \%, p=0.057$ ) compared to END. Higher disease-specific survival (DSS) rates were found for pT1 SLNB patients compared to pT1 END patients (96\% vs. $90 \%, p=0.048$ ) [19]. In the French Senti-MER trial, the only randomized clinical trial, 307 cT1-2N0 were randomized between END and SNLB. After a mean follow-up of almost 5 years, the 2- and 5-year neck node recurrence free survival for END and SLNB were comparable: $89.6 \%$ vs. $90.7 \%$ and $89.6 \%$ vs. $89.4 \%$, respectively. Also, locoregional free, disease-specific and overall survival were not different for both groups. Lower morbidity was observed in the SLNB arm during the first year post-surgery [20]. See also Table 1.

Several studies reported the differences in complication rates, postoperative morbidity and cost-effectiveness in favor of the SLNB compared to the END procedures [9, 21-27]. Moya-Plana et al. found a significant higher complication rate for patients after neck dissection as compared to SNB only patients: $28 \%$ vs. $8 \%(p<0.0001)$ presented at least one complication [9]. Using different scoring systems, 
Murer et al. found a higher shoulder morbidity for END as compared to SNLB [23]. Govers et al. performed a modeling study of the cost-effectiveness and found SLNB to be the most cost effective strategy for diagnosing and treating OSCC patients [29].

Due to the overall predominantly good performance, SLNB was quickly adopted during the last decade as diagnostic staging method and is now incorporated in many national guidelines including those from The Netherlands, United Kingdom (NICE) and USA (NCCN). Compared to the routinely performed END, its minimally invasive design combined with a high sensitivity for detecting occult metastases offers the possibility to reduce the number of surgically overtreated patients. Although SLNB for cT1-2N0 OSCC is also associated with reduced morbidity, costs and length of hospital stay, increased quality of life and equivalent overall survival compared with END it remains rarely used in the United States [18]. Contrary, in Europe, SLNB is increasingly used in cT1-2N0 OSCC patients. A survey in the Netherlands revealed that in 2006 none of the head and neck centers used SLNB routinely [28], while in 2014, four centers [29] and nowadays, seven of the eight major centers of the Dutch Head and Neck Society (NWHHT) use SLNB routinely in cT1-2N0 OSCC patients.

\section{Improved staging by sentinel lymph node biopsy}

The SLNB procedure can assess the individual drainage pattern. In addition, despite some variability among different studies, most histological protocols for the evaluation of SLNB typically involves consist of sectioning of the lymph node at $2 \mathrm{~mm}$ thickness, subsequent multiple levels and the use immunohistochemistry for cytokeratin. This approach allows for the identification of isolated tumor cells and micrometastases. Such histological protocols would be impractical for a large specimen derived from an END. In this way, one could argue that SLNB provides better results in the detection of lymph node metastasis and therefore improved staging as compared to END with routine histopathological examination.

Several studies showed a significant difference between SLNB-positive and SLNB-negative patients. Broglie et al. found an overall survival (OS), disease specific survival (DSS) and disease-free survival (DFS) at 3 years for SLNBnegative and SLNB-positive patients of $98 \%$ and $71 \%, 95 \%$ and $76 \%$, and $98 \%$ and $73 \%$, respectively [6]. In a study of Den Toom et al. OS and DFS for SLNB negative were $100 \%$ and $84 \%$ compared to $73 \%$ and $88 \%$ for SLNB-positive patients, respectively [7]. This significant difference for OS was also found in the SENT trial [14].
Broglie et al. were the first to demonstrate that even small tumor deposits only detectable by the extensive histopathologic workup of the SLNB protocol have a significant impact on tumor control and survival in early OSCC. Forty-nine of 111 patients (38\%) had positive SLNs, 10 had ITCs, 19 had micrometastases, and 13 had macrometastases. They found a statistically significant difference between the SLNnegative group and ITCs, micrometastases and macrometastases groups in different survival estimates [7]. Pedersen et al. found also a shorter DFS for patients with ITC or micrometastases compared patients without metastases [8]. In the SENT trial a significant difference was found for OS between ITC, micrometastasis and macrometastasis [14]. These data underline the clinical importance of detecting ITCs and micrometastasis in OSCC.

This means that the presence of positive SLNs and size of tumor deposits within these SLNs, i.e. ITCs, micrometastases and macrometastases, may be used to predict survival and individualized treatment planning. As it is not feasible to investigate all lymph nodes in an END specimen by step serial sectioning and immunohistochemistry, SLNB will more accurately stage the neck enabling better prognostication and more individualize additional treatment.

On the other hand, free soft tissue disease (defined as a metastatic carcinoma in the soft tissue of the neck with no evidence of a lymph node architecture) can probably not be detected by SLNB [30].

\section{Contralateral neck}

SLNB stages both the ipsilateral as well as the contralateral neck in lateralized cT1-2N0 OSCC patients, whereas the contralateral neck is usually not addressed by END in cT1$2 \mathrm{~N} 0$ OSCC not involving the midline. Although the reported incidence of contralateral lymph node metastases in lateralized early-stage OSCC is relatively low, underdiagnosis of the contralateral clinically negative neck is undesirable, especially since the presence of contralateral lymph node metastases from OSCC have been associated with poor DSS [31]. Contralateral lymphatic drainage and positive SLNs have been reported in several studies. Moya-Plana et al. found among 195 unilateral primary tumors contralateral SLNs in $12.8 \%$ [9]. In the Dutch SNUS trial, contralateral drainage was observed in $13 \%$ of well-lateralized tumors [13]. In the European SENT trial, unilateral drainage only was found in $40 \%$ of midline OSCC and contralateral drainage in $12.4 \%$ of well-lateralized OSCC [14]. Mølstrøm et al. investigated the topographical distribution of SLNs and metastases from cT1-T2N0 OSCC. In $28.5 \%$ of patients with midline tumors, only unilateral lymphatic drainage on lymphoscintigraphy was observed. Patients with lateralized OSCC had unexpected bilateral or contralateral drainage patterns in $22.6 \%$ and contralateral positive SLNs in $9 \%$, 
which would have been missed by elective neck dissection of the ipsilateral neck [11].

\section{Previously treated neck}

Patients with a history of surgery or radiotherapy in the neck may have aberrant lymphatic drainage caused by disruption of lymphatic channels. Therefore, elective treatment of the same levels at risk as in the primary setting may not be appropriate. SNB in the pretreated neck appears to be feasible and renders an assessment of the individual lymphatic drainage pattern, compensating for a potential variability after previous treatment of the neck. Unexpected lymphatic drainage patterns (first SLN at level IV or V and contralateral neck in well-lateralized tumors) were found in 30-67\% of cT1-2N0 OSCC patients with a previously treated neck. SNLB was able to upstage the neck of these patients in $7 \%$ $[32,33]$.

Therefore, SLNB can be used to assess individual and unexpected lymphatic drainage patterns allowing for tailoring treatment of the neck reducing under- and overtreatment, also in a pretreated neck.

\section{Selection of patients for sentinel lymph node biopsy}

Traditionally SLNB in OSCC patients is reserved for tumors which can be transorally resected without opening the neck for resection of the primary tumor or reconstruction and a clinically negative neck. In the 7th edition of the TNM classification these tumors were generally staged cT1-2N0; however, in the 8th edition, the depth of invasion is also taken into consideration. This results in upstaging $\mathrm{T} 2$ tumors with deep invasion $(>10 \mathrm{~mm}$ ) to T3 tumors, which may be still suitable for resection via transoral approach.

\section{Depth of invasion}

Some institutes use depth of invasion for the decision to perform an END or watchful waiting and continued this for a decision to perform SLNB or END. Although depth of invasion (DOI) is associated with risk of lymph node metastases and survival a cut-off DOI for SLNB or END is difficult to determine. Den Toom et al. investigated if extent of DOI can predict occult nodal disease in 199 patients with cT1-2N0 (7th TNM) OSCC staged by SNLB. The mean DOI of patients with and without lymph node metastasis was significantly different: $6.6 \mathrm{~mm}$ vs. $4.7 \mathrm{~mm}(p=0.003)$. The ROC showed an area under the curve of 0.65 with a most optimal cut-off point of $3.4 \mathrm{~mm}$ DOI (sensitivity $83 \%$ and specificity $47 \%$ ). However, regional metastases were still found in $15 \%$ of patients with DOI $\leq 3.4 \mathrm{~mm}$ and it was concluded that DOI seems to be an insufficient predictor to assess which cT1-2N0 OSCC patients can refrain from SLNB. Therefore, it was suggested that staging of the neck using SLNB in early-stage oral cancer patients should also be performed in tumors with limited DOI and probably in T3 OSCC $\leq 4 \mathrm{~cm}$ diameter (thus tumors upstaged from T1-2 to T3 by 8th TNM) [34]. An exception is minimally invasive lesions with less than one mm depth of invasion, where lymphatic metastases are exceedingly rare. These are lesions that are often initially diagnosed as carcinoma in situ, and later minimal invasion is found on the resection [35].

\section{Floor of mouth}

Generally, SLNB has proven to reliably stage the clinically negative neck in early-stage OSCC with high sensitivity and negative predictive value. However, in floor of mouth OSCC, the accuracy to detect occult lymph node metastasis is significantly lower. In a series of 488 cT1-2N0 OSCC patients, SLNB had a lower sensitivity in FOM tumors than in non-FOM locations: $63 \%$ and $86 \%(p=0.008)$, respectively [19].

When SNLs are located in close vicinity of the tracer injection site, due to the limited resolution of the $\gamma$-camera and SPECT, the hotspot of the tracer injection site can hide adjacent SLNs. This 'shine-through phenomenon' hampers discrimination between tracer injection site and SLNs. A lower accuracy for SLNB can result in missing occult lymph node metastasis, which will inevitably develop into clinical manifestation of disease and consequently induce a poor oncological prognosis.

There are some indications that this shine through phenomenon explains the lower sensitivity of SLNB in FOM OSCC in the literature. In the series of Pedersen et al., six of the nine false negative SLNB procedures were in FOM tumors and six of these nine regional recurrences were upstream from the levels initially explored (four in level IA; two in level IB) [8]. In the study of Moya-Plana et al., 6 of the $46(13 \%)$ patients with a positive SLNB, SLNs were positive in II-III, and positive lymph nodes were found in IB area upon completion neck dissection [9]. In the study of Den Toom et al. in 7 of the 11 (64\%), FOM patients with a false negative SLNB developed a regional recurrence in level I [7].

These findings suggest that in FOM OSCC occult metastasis are frequently missed by SLNB due to the shine through phenomenon and, therefore, the use of the standard SLNB technique in FOM OSCC is debatable. To overcome this limitation of SLNB in FOM OSCC, a superselective neck dissection of the preglandular triangle of level I was described by Stoeckli et al. and is now routinely used in some centers. They evaluated this technique in 40 consecutive and prospectively enrolled FOM OSCC patients. Eleven 
of $22(50 \%)$ SLNS were only detected intraoperatively. Using this technique in addition to the standard technique a false negative rate of $8.3 \%$ and negative predictive value of $96.4 \%$ were reported [36]. Therefore, it is advised not to perform SLNB routinely or do a superselective neck dissection of the preglandular triangle of level I in FOM OSCC until the SNLB procedure is improved.

\section{Techniques for improving sentinel lymph node biopsy accuracy}

Technical developments are needed to bring the diagnostic accuracy of SLNB for early-stage OSCC to a higher level, particularly for FOM tumors. Some techniques which improve SNLB are already implemented in current routine SLNB, while other techniques are still under investigation.

\section{Single photon emission computed tomography with computed tomography}

Historically, visualization of SLNs is routinely carried out with static and dynamic planar lymphoscintigraphy. SPECT/ CT was already introduced in oral cancer in 2003 [37]. Although most studies concluded that SPECT/CT improves localization of the SLNs and detection of additional SLNs [38], it took some years to become part of the standard lymphoscintigraphy protocol [39]. SPECT-CT has especially advantages for tumors with close proximity to the SLN and regions with complex lymphatic drainage which is the case in OSCC, particularly FOM tumors [40]. Den Toom et al. investigated the additional value of SPECT/CT to planar images for the identification SLNs in 66 patients with earlystage OSCC. SPECT-CT identified 15 additional SLNs in 14 patients (22\%). In $13 \%$ of these additional SLNs, the only metastasis was found, resulting in an upstaging rate of $3 \%$. In $20 \%$ of the patients with at least one positive SLN, the only positive SLN was detected by the addition of SPECT/ CT. SPECT/CT was considered to add important anatomical information in two patients (3\%) [41]. The addition of SPECT/CT to planar lymphoscintigraphy is now recommended for the identification of more (positive) SLNs and better topographical orientation for surgery in SLNB for early-stage OSCC [42].

\section{Fluorescence imaging}

For intraoperative visualization of SLNs, traditionally peritumoral injection of blue dye has been traditionally used. Because of its very limited additional value [43], the use of blue dye in SLNB was abandoned by many. Near-infrared (NIR) fluorescence imaging is nowadays increasingly used in SLNB. NIR dyes have the advantage to exhibit reasonable tissue penetration of excited and emitted light with negligible auto-fluorescence, resulting in high target-to-background contrast. NIR fluorescence imaging provides high-resolution images which can be obtained in real time during the surgical procedure, even if the structure of interest is covered by some tissue (in contrast to blue dye). Another advantage of NIR fluorescence imaging is that it is much better suited for detection of SLNs close to the primary, because there is negligible influence of fluorescence signal coming from the injection site given the limited penetrance of the emitted light signal through surrounding tissue. Because of its easy availability indocyanin green (ICG) is the most frequently used NIR-fluorescent compound. Because ICG alone has a poor retention in SLN it is combined with nanocolloidal albumin. Van den Berg et al. were the first to evaluate the added value of intraoperative fluorescence imaging to the conventional radioguided procedure in 14 OSCC patients using ICG- ${ }^{99 \mathrm{~m}} \mathrm{Tc}$-nanocolloid, a hybrid tracer that is both radioactive and fluorescent. SLNs were detected preoperatively by lymphoscintigraphy, including SPECT-CT, and intraoperatively with a gamma-probe and NIR camera. In four patients, a SLN located close to the primary injection site could only be intraoperatively localized using fluorescence imaging. In one patient this SLN contained a metastasis. In 4 of the 14 OSCC patients where the SLN was located close to the primary injection site the SLN could only be localized by fluorescence imaging [44]. In a study of Christensen et al. in 30 OSCC patients using the hybrid tracer ICG- ${ }^{99 \mathrm{~m}}$ Tc-nanocolloid SLNB revealed a total of 94 SLNs of which $11(12 \%)$ could only be identified by NIR. The majority of those were located in level I close to the primary tumor [45]. The combination of ICG and a radiopharmaceutical enables the identification of SLNs more easily and rapidly than using a radiopharmaceutical alone. Intraoperative fluorescence guidance seems of particular value when SLNs are located in close proximity to the injection site. Other tracers with improved optical properties have been tested in preclinical settings [46, 47].

\section{Novel tracers}

Radiolabelled tracers other than colloidal albumin with other characteristics may improve intraoperative differentiation between SLN and injection site [48]. A new radioactive agent, ${ }^{99 \mathrm{~m}} \mathrm{Tc}$-tilmanocept (Lymphoseek ${ }^{\circledR}$, Navidea Biopharmaceuticals, Inc.), has been specifically designed for SLN identification and is registered for this purpose in both the USA and Europe. ${ }^{99 \mathrm{~m}} \mathrm{Tc}$-tilmanocept is a small sized receptor (CD206) targeted SLN detection agent. Due to its proposed rapid clearance from the injection site, rapid uptake and high retention within the SLN, as well as low uptake by the remaining (higher echelon) lymph nodes, ${ }^{99 \mathrm{~m}} \mathrm{Tc}$-tilmanocept may particularly be of benefit in floor of 
mouth tumors and other head and neck tumors with complex drainage patterns and close spatial relation to the SLNs [49].

A multicenter validation study using ${ }^{99 \mathrm{~m}} \mathrm{Tc}$-tilmanocept for SLNB in head and neck squamous cell carcinoma of skin and (mainly) oral cavity showed an SLN identification rate of $97.6 \%$, a false negative rate of $2.56 \%$ and an NPV of $97.8 \%$. Of note, these favorable outcomes were also obtained in FOM cancers, which strengthened the idea that ${ }^{99 \mathrm{~m}}$ Tc-tilmanocept may diminish the shine through effect and improve the SLN detection rate for this subsite [50].

A recent prospective within-patient evaluation study compared ${ }^{99 \mathrm{mTc}} \mathrm{Tc}$-tilmanocept with ${ }^{99 \mathrm{~m}} \mathrm{Tc}$-nanocolloid for SLN detection in 20 patients with early-stage OSCC, who underwent lymphoscintigraphy with both tracers. ${ }^{99 \mathrm{~m}} \mathrm{Tc}-$ tilmanocept had a higher injection site clearance, but at the same time, a lower uptake in the SLN, resulting in a comparable SLN to injection site ratio, using ${ }^{99 \mathrm{~m}} \mathrm{Tc}$-nanocolloid [51]. ${ }^{99 \mathrm{~m}} \mathrm{Tc}$-tilmanocept is registered at the FDA and EMA using an activity of $74 \mathrm{MBq}$, which is lower than activities routinely used with ${ }^{99 \mathrm{~m}} \mathrm{Tc}$-nanocollidal tracers. The low radioactivity used results in relatively low uptake in SLNs of ${ }^{99 \mathrm{~m}} \mathrm{Tc}$-tilmanocept which may limit intraoperative detection of SLNs. This might be overcome by a higher injection activity. Larger trials, preferably multicenter randomized clinical trials, are needed to determine if ${ }^{99 \mathrm{~m}} \mathrm{Tc}$-tilmanocept can improve SLNB in OSCC patients.

Several other techniques are currently under development in OSCC patients. These techniques include the use of PETCT, CT lymphography, MRI lymphography using contrast agents or superparamagnetic iron oxide, contrast-enhanced ultrasound mapping using microbubbles, and freehand SPECT [52].

\section{Subsequent treatment after positive sentinel lymph node biopsy}

Because there is no reliable method for detection or prediction of non-SLN metastasis, patients with positive SLNB undergo a subsequent (completion) neck treatment. In an analysis of the reported data in the literature, a relation between the size of tumor deposits in the SLN and the risk of a non-SLN metastasis in the completion neck dissection was found: $13 \%$ for ITC, $20 \%$ for micrometastasis and $40 \%$ for macrometastasis [53]. Since in patients with ITC in a SLN has a substantial probability of non-SLN metastasis in the neck, treatment of the neck remains mandatory after any category of positive SLNB in early-stage OSCC patients.

\section{Subsequent neck dissection versus radiotherapy}

Both neck dissection and radiotherapy can treat eventual non-SLN lymph node metastasis well. The choice of definitive treatment modality of the neck after positive SLNB is highly dependent on the treatment modality needed for adjuvant treatment of the primary tumor and eventual previous treatment of the neck.

In a multicenter retrospective study of 107 OSCC patients with positive SLNB ITCs were detected as the largest metastatic deposit in SLNB in 15 patients (14\%), micrometastasis was detected in 31 patients (29\%) and macrometastasis in 61 patients (57\%). A positive SLNB was followed by an additional (selective) neck dissection in $86 \%$ of the patients). Ten patients received radiotherapy on the neck and remained free of regional recurrence. Five patients refused any additional treatment [19]. In a study of Pedersen et al., the overall neck control rate in early-stage OSCC patients who underwent SLNB was $96 \%$ ( 243 of 253 patients). The regional recurrence rates for the 68 SLNB-positive patients who underwent subsequent neck dissection $(n=36$ of whom 7 with adjuvant radiotherapy), patients who received radiotherapy only $(n=19)$ and patients were received no additional treatment and subsequently underwent close clinical follow-up $(\mathrm{n}=13)$ were $11 \%, 16 \%$ and $31 \%$, respectively [8].

From these studies, it may be concluded that the first choice for the treatment of the neck after positive SLNB is neck dissection; however, definitive radiotherapy is probably a reliable alternative.

\section{Extent of neck disssection}

A report of the European multicenter SENT study on 109 OSCC patients with positive SLNB showed additional (nonSLN) metastases in $34.4 \%$ of the neck dissection specimens. The risk of non-SLN metastases outside the adjacent basins of the positive SLN was low (7.1\%) [54]. In a report on the 3-year results of the SENT study 94 patients with a positive SLNB who underwent subsequent neck dissection were analyzed. In $85 \%$ of cases, no further positive nodes were found in the completion specimen. Of the 15 patients with additional positive non-SLN, 13 (87\%) were located in the same neck level as the SLN or an adjacent neck level [14]. In another study of 36 OSCC patients with positive SLNB, all non-SLN metastases were found in levels I-IV except for one in level V. In this latter patient, two positive SLNs and five additional non-SLNs metastases were found. In four of the six $(67 \%)$ patients with non-SLN metastasis, these were only found in nonadjacent levels [53]. Pedersen et al. analyzed 36 OSCC patients with positive SNLB who underwent subsequent neck dissection. Additional lymph node macrometastases were histopathologically identified in only 2 of the 36 patients [8]. In a study on the topographical distribution of SLNs and non-SLN metastases in 220 patients with early-stage OSCC and lymph node metastases, 53 patients had positive SLNB and underwent subsequent 
neck dissection. Metastatic involvement of neck level IV was rare and only observed in two patients with anterior tongue cancer. No patients had level V involvement [11].

The results of these studies support the use of a selective neck dissection (levels I-III/IV), in cases of a SLNB-positive neck in whom additional neck dissection is indicated. If future larger studies report more specifically on the level involved by non-SLN metastases more tailored (super)selective neck dissections may be defined.

\section{Guidelines}

In 2009, joint practice guidelines for radionuclide lymphoscintigraphy in early-stage OSCC were published to outline the at that time best practice guidelines for SLN localization in OSCC. These guidelines were prepared by a multidisciplinary expert panel of surgeons, nuclear physicians and pathologists under the joint auspices of the European Association of Nuclear Medicine (EANM) Oncology Committee and the Sentinel European Node (SENT) Trial Committee [55]. In 2018, the eighth international symposium for sentinel node biopsy in head and neck cancer was held. This consensus conference aimed to update the multidisciplinary SLNB guidelines for nuclear medicine, surgery and pathology in early-stage OSCC. Recently, the updated EANM practical guidelines for sentinel lymph node localization in OSCC were published [42]. Also, recently surgical consensus guidelines on SLNB in patients with OSCC were reported [56]. Unfortunately, consensus was not achieved in all areas, highlighting the need for more research on SLNB in OSCC. Large registries may be helpful to further improve these guidelines in the near future.

\section{Limitations}

This review focuses on SLNB in clinical practice of earlystage OSCC patients. In this review, only results of many studies on different topics are described. Some recent metaanalysis $[16,17]$ on the diagnostic accuracy of SLNB are mentioned. Moreover, the only randomized clinical trial comparing SLNB and END, which is very recently reported, is included. For other topics, data are limited and probably too heterogeneous to allow for high-quality meta-analysis. Moreover, only routinely used procedures are described, while many promising new developments, e.g. MR lymphography, CT lymphography, PET lymphoscintigraphy and contrast-enhanced lymphosonography, are under investigation to improve SLNB [57]. SLNB is not routinely used in other head and neck sites, because most of these tumors are treated non-surgically, i.e. radiotherapy with or without chemotherapy, and peri-tumoral injections are more difficult to perform than in the oral cavity, e.g. larynx [58, 59]. However, using these techniques, the SLNB procedure can also be used for individualized prophylactic neck irradiation [60, 61]. Although SLNB is a reliable technique, it is good to realize that it has also some limitations: SLNB is an invasive technique and since no intraoperative reliable technique to examine the SLNB is yet available, an eventual subsequent neck dissection has to be performed in a second-stage procedure [62].

\section{Conclusion}

In conclusion, detection of lymph node metastases in earlystage OSCC using SLNB is a good alternative for END, except for floor of mouth tumors. SLNB potentially stages the neck more accurately enabling better prognostication and more individualized additional treatment. The main limitations of SLNB are the invasive and complex procedure and in SLNB-positive patients a subsequent neck dissection as second-stage procedure. Several techniques, e.g. MR lymphography, CT lymphography, PET lymphoscintigraphy and contrast-enhanced lymphosonography, are currently under development in OSCC patients with the aim to improve the diagnostic accuracy of SLNB for OSCC, particularly for FOM tumors, and to allow the use of SNLB for other purposes, e.g. individualized prophylactic neck irradiation. Treatment of the neck, although limited to levels I-IV, remains mandatory after any category of positive SLNB.

\section{Compliance with ethical standards}

Conflict of interest The authors declare that they have not conflict of interest.

Open Access This article is licensed under a Creative Commons Attribution 4.0 International License, which permits use, sharing, adaptation, distribution and reproduction in any medium or format, as long as you give appropriate credit to the original author(s) and the source, provide a link to the Creative Commons licence, and indicate if changes were made. The images or other third party material in this article are included in the article's Creative Commons licence, unless indicated otherwise in a credit line to the material. If material is not included in the article's Creative Commons licence and your intended use is not permitted by statutory regulation or exceeds the permitted use, you will need to obtain permission directly from the copyright holder. To view a copy of this licence, visit http://creativecommons.org/licenses/by/4.0/.

\section{References}

1. De Bree R, Takes RP, Castelijns JA et al (2015) Advances in diagnostic modalities to detect occult lymph node metastases in head and neck squamous cell carcinoma. Head Neck 37:1829-1839 
2. De Bree R, Takes RP, Shah JP et al (2019) Elective neck dissection in oral squamous cell carcinoma: past, present and future. Oral Oncol 90:87-93

3. De Bree R, Nieweg OE (2015) The history of sentinel node biopsy in head and neck cancer: from visualization of lymphatic vessels to sentinel nodes. Oral Oncol 51:819-823

4. Civantos FJ, Stoeckli SJ, Takes RP et al (2010) What is the role of sentinel lymph node biopsy in the management of oral cancer in 2010? Eur Arch Otorhinolaryngol 267:839-844

5. Rinaldo A, Devaney KO, Ferlito A (2004) Immunohistochemical studies in the identification of lymph node micrometastases in patients with squamous cell carcinoma of the head and neck. ORL J Otorhinolaryngol Relat Spec 66:38-41

6. Broglie MA, Haerle SK, Huber GF, Haile SR, Stoeckli SJ (2013) Occult metastases detected by sentinel node biopsy in patients with early oral and oropharyngeal squamous cell carcinomas: impact on survival. Head Neck 35:660-666

7. Den Toom IJ, Heuveling DA, Flach GB et al (2015) Sentinel node biopsy for early-stage oral cavity cancer: the VU University Medical Center experience. Head Neck 37:573-578

8. Pedersen NJ, Jensen DH, Hedbäck N et al (2016) Staging of early lymph node metastases with the sentinel lymph node technique and predictive factors in $\mathrm{T} 1 / \mathrm{T} 2$ oral cavity cancer: a retrospective single-center study. Head Neck 38(Suppl 1):E1033-1040

9. Moya-Plana A, Aupérin A, Guerlain J et al (2018) Sentinel node biopsy in early oral squamous cell carcinomas: long-term followup and nodal failure analysis. Oral Oncol 82:187-194

10. Vorburger MS, Broglie MA, Soltermann A et al (2012) Validity of frozen section in sentinel lymph node biopsy for the staging in oral and oropharyngeal squamous cell carcinoma. J Surg Oncol 106:816-819

11. Mølstrøm J, Grønne M, Green A, Bakholdt V, Sørensen JA (2019) Topographical distribution of sentinel nodes and metastases from T1-T2 oral squamous cell carcinomas. Eur J Cancer 107:86-92

12. Boeve K, Schepman KP, Schuuring E et al (2018) High sensitivity and negative predictive value of sentinel lymph node biopsy in a retrospective early stage oral cavity cancer cohort in the Northern Netherlands. Clin Otolaryngol. https://doi.org/10.1111/coa.13107 (Online ahead of print)

13. Flach GB, Bloemena E, Klop WM et al (2014) Sentinel lymph node biopsy in clinically N0 T1-T2 staged oral cancer: the Dutch multicenter trial. Oral Oncol 50:1020-1024

14. Schilling C, Stoeckli SJ, Haerle SK et al (2015) Sentinel European Node Trial (SENT): 3-year results of sentinel node biopsy in oral cancer. Eur J Cancer 51:2777-2784

15. Civantos FJ, Zitsch RP, Schuller DE et al (2010) Sentinel lymph node biopsy accurately stages the regional lymph nodes for T1-T2 oral squamous cell carcinomas: results of a prospective multiinstitutional trial. J Clin Oncol 28:1395-1400

16. Liu M, Wang SJ, Yang X, Peng H (2017) Diagnostic efficacy of sentinel lymph node biopsy in early oral squamous cell carcinoma: a meta-analysis of 66 studies. PLoS ONE 12:e0170322

17. Yang Y, Zhou J, Wu H (2017) Diagnostic value of sentinel lymph node biopsy for cT1/T2N0 tongue squamous cell carcinoma: a meta-analysis. Eur Arch Otorhinolaryngol 274:3843-3852

18. Cramer JD, Sridharan S, Ferris RL, Duvvuri U, Samant S (2019) Sentinel lymph node biopsy versus elective neck dissection for Stage I to II oral cavity cancer. Laryngoscope 129:162-169

19. Den Toom IJ, Boeve K, Lobeek D et al (2020) Elective neck dissection or sentinel lymph node biopsy in early stage oral cavity cancer patients: the dutch experience. Cancers (Basel) 12:1783

20. Garrel R, Poissonnet G, Moyà Plana A et al (2020) Equivalence randomized trial to compare treatment on the basis of sentinel node biopsy versus neck node dissection in operable T1T2N0 oral and oropharyngeal cancer. J Clin Oncol. https://doi. org/10.1200/JCO.20.01661 (Online ahead of print)
21. Hernando J, Villarreal P, Alvarez-Marcos F, Gallego L, GarcíaConsuegra L, Junquera L (2014) Comparison of related complications: sentinel node biopsy versus elective neck dissection. Int J Oral Maxillofac Surg 43:1307-1312

22. Schiefke F, Akdemir M, Weber A, Akdemir D, Singer S, Frerich B (2009) Function, postoperative morbidity, and quality of life after cervical sentinel node biopsy and after selective neck dissection. Head Neck 31:503-512

23. Murer K, Huber GF, Haile SR (2010) Stoeckli SJ (2010) Comparison of morbidity between sentinel node biopsy and elective neck dissection for treatment of the n 0 neck in patients with oral squamous cell carcinoma. Head Neck 33:1260-1264. https://doi. org/10.1002/hed.21622

24. Hernando J, Villarreal P, Álvarez MF, Consuegra LG, Gallego L, Junquera L (2016) Sentinel node biopsy versus elective neck dissection. Which is more cost-effective? A prospective observational study. J. Cranio-Maxillofacial Surg 33:1260-1264

25. Govers TM, Takes RP, Baris Karakullukcu M et al (2013) Management of the N0 neck in early stage oral squamous cell cancer: a modeling study of the cost-effectiveness. Oral Oncol 49:771-777

26. van der Linden N, Flach GB, de Bree R, Uyl-de Groot CA (2016) Cost-utility of sentinel lymph node biopsy in cT1-T2N0 oral cancer. Oral Oncol 53:20-26

27. O'Connor R, Pezier T, Schilling C, McGurk M (2013) The relative cost of sentinel lymph node biopsy in early oral cancer. J Craniomaxillofac Surg 41:721-727

28. De Bree R, van der Waal I, Doornaert P, Werner JA, Castelijns JA, Leemans CR (2009) Indications and extent of elective neck dissection in patients with early stage oral and oropharyngeal carcinoma: nationwide survey in The Netherlands. J Laryngol Otol 123:889-898

29. Govers TM, de Kort TB, Merkx MA et al (2016) An international comparison of the management of the neck in early oral squamous cell carcinoma in the Netherlands, UK, and USA. J Craniomaxillofac Surg 44:62-69

30. Devaney KO, Rinaldo A, Rodrigo JP, Ferlito A (2006) Sentinel node biopsy and head and neck tumors-where do we stand today? Head Neck 28:1122-1131

31. Feng Z, Niu LX, Yuan Y, Peng X, Guo CB (2014) Risk factors and treatment of contralateral neck recurrence for unilateral oral squamous cell carcinoma: a retrospective study of 1482 cases. Oral Oncol 50:1081-1088

32. Flach GB, Broglie MA, van Schie A et al (2012) Sentinel node biopsy for oral and oropharyngeal squamous cell carcinoma in the previously treated neck. Oral Oncol 48:85-89

33. Den Toom IJ, Boeve K, van Weert S et al (2019) High rate of unexpected lymphatic drainage patterns and a high accuracy of the sentinel lymph node biopsy in oral cancer after previous neck treatment. Oral Oncol 94:68-72

34. Den Toom IJ, Janssen LM, van Es RJJ et al (2019) Depth of invasion in patients with early stage oral cancer staged by sentinel node biopsy. Head Neck 41:2100-2106

35. Mohit-Tabatabai MA, Sobel HJ, Rush BF, Mashberg A (1986) Relation of thickness of floor of mouth stage I and II cancers to regional metastasis. Am J Surg 152:351-353

36. Stoeckli SJ, Huebner T, Huber GF, Broglie MA (2016) Technique for reliable sentinel node biopsy in squamous cell carcinomas of the floor of mouth. Head Neck 38:1367-1372

37. Even-Sapir E, Lerman H, Lievshitz G et al (2003) Lymphoscintigraphy for sentinel node mapping using a hybrid SPECT/CT system. J Nucl Med 44:1413-1420

38. Stephan KH, Sandro JS (2011) SPECT/CT for lymphatic mapping of sentinel nodes in early squamous cell carcinoma of the oral cavity and oropharynx. Int J Mol Imaging 106068 
39. Tartaglione G, Stoeckli SJ, de Bree R et al (2016) Sentinel node in oral cancer: the nuclear medicine aspects. A survey from the sentinel European Node Trial. Clin Nucl Med 41:534-542

40. Vermeeren L, van der Ploeg IM et al (2010) SPECT/CT for preoperative sentinel node localization. J Surg Oncol 101:184-190

41. Den Toom IJ, van Schie A, van Weert S et al (2017) The added value of SPECT-CT for the identification of sentinel lymph nodes in early stage oral cancer. Eur J Nucl Med Mol Imaging 44:998-1004

42. Giammarile F, Schilling C, Gnanasegaran G et al (2019) The EANM practical guidelines for sentinel lymph node localisation in oral cavity squamous cell carcinoma. Eur J Nucl Med Mol Imaging 46:623-637

43. Ross G, Shoaib T, Soutar DS et al (2002) The use of sentinel node biopsy to upstage the clinically N0 neck in head and neck cancer. Arch Otolaryngol Head Neck Surg 128:1287-1291

44. Van den Berg NS, Brouwer OR, Klop WM et al (2012) Concomitant radio- and fluorescence-guided sentinel lymph node biopsy in squamous cell carcinoma of the oral cavity using ICG-(99m) Tc-nanocolloid. Eur J Nucl Med Mol Imaging 39:1128-1136

45. Christensen A, Juhl K, Charabi B et al (2016) Feasibility of real-time near-infrared fluorescence tracer imaging in sentinel node biopsy for oral cavity cancer patients. Ann Surg Oncol 23:565-572

46. Heuveling DA, Visser GW, de Groot M et al (2012) Nanocolloidal albumin-IRDye $800 \mathrm{CW}$ : a near-infrared fluorescent tracer with optimal retention in the sentinel lymph node. Eur J Nucl Med Mol Imaging 39:1161-1168

47. Qin Z, Hoh CK, Hall DJ, Vera DR (2015) A tri-modal molecular imaging agent for sentinel lymph node mapping. Nucl Med Biol 42:917-922

48. Vidal-Sicart S, Vera DR, Valdes Olmos RA (2018) Next generation of radiotracers for sentinel lymph node biopsy: what is still necessary to establish new imaging paradigms? Rev Esp Med Nucl Imagen Mol 37:373-379

49. Wallace AM, Hoh CK, Vera DR, Darrah DD, Schulteis G (2003) Lymphoseek: a molecular radiopharmaceutical for sentinel node detection. Ann Surg Oncol 10:531-538

50. Agrawal A, Civantos FJ, Brumund KT et al (99m)Tc]Tilmanocept Accurately Detects Sentinel Lymph Nodes and Predicts Node Pathology Status in Patients with Oral Squamous Cell Carcinoma of the Head and Neck: Results of a Phase III Multi-institutional Trial. Ann Surg Oncol 22:3708-3715

51. Den Toom IJ, Mahieu R, van Rooij R et al (2020) Sentinel lymph node detection in oral cancer: a within-patient comparison between [99m Tc]Tc-tilmanocept and [99m Tc]Tc-nanocolloid. Eur J Nucl Med Mol Imaging. https://doi.org/10.1007/s0025 9-020-04984-8 (Online ahead of print)
52. De Bree R, Dankbaar JW, de Keizer B (2019) New Developments in Sentinel Lymph Node Biopsy Procedure in Localized Oral Cancer. JAMA Otolaryngol Head Neck Surg. https://doi.org/10.1001/ jamaoto.2019.1342 (Online ahead of print)

53. Den Toom J, Bloemena E, van Weert S, Karagozoglu KH, Hoekstra OS, de Bree R (2017) Additional non-sentinel lymph node metastases in early oral cancer patients with positive sentinel lymph nodes. Eur Arch Otorhinolaryngol 274:961-968

54. Gurney BA, Schilling C, Putcha V et al (2012) Implications of a positive sentinel node in oral squamous cell carcinoma. Head Neck 34:1580-1585

55. Alkureishi LW, Burak Z, Alvarez JA et al (2009) Joint practice guidelines for radionuclide lymphoscintigraphy for sentinel node localization in oral/oropharyngeal squamous cell carcinoma. Eur J Nucl Med Mol Imaging 36:1915-1936

56. Schilling C, Stoeckli SJ, Vigili MG et al (2019) Surgical consensus guidelines on sentinel node biopsy (SNB) in patients with oral cancer. Head Neck 41:2655-2664

57. Mahieu R, de Maar JS, Nieuwenhuis ER et al (2020) New developments in imaging for sentinel lymph node biopsy in early-stage oral cavity squamous cell carcinoma. Cancers (Basel) 12(10):3055

58. Lawson G, Matar N, Nollevaux MC et al (2010) Reliability of sentinel node technique in the treatment of N0 supraglottic laryngeal cancer. Laryngoscope 120:2213-2217

59. Flach GB, Bloemena E, van Schie A et al (2013) Sentinel node identification in laryngeal cancer: feasible in primary cancer with previously untreated neck. Oral Oncol 49:165-216

60. Longton E, Lawson G, Bihin B, Mathieu I, Hanin FX, Deheneffe S, Vander Borght T, Laloux M, Daisne JF et al (2020) Individualized prophylactic neck irradiation in patients with $\mathrm{cNO}$ head and neck cancer based on sentinel lymph node(s) identification: definitive results of a prospective Phase 1-2 study. Int J Radiat Oncol Biol Phys 107:652-661

61. de Veij Mestdagh PD, Walraven I, Vogel WV et al (2020) SPECT/ CT-guided elective nodal irradiation for head and neck cancer is oncologically safe and less toxic: a potentially practice-changing approach. Radiother Oncol 147:56-63

62. Bluemel C, Rubello D, Colletti PM et al (2015) Sentinel lymph node biopsy in oral and oropharyngeal squamous cell carcinoma: current status and unresolved challenges. Eur J Nucl Med Mol Imaging 42:1469-1480

Publisher's Note Springer Nature remains neutral with regard to jurisdictional claims in published maps and institutional affiliations. 